\title{
3D Shape Analysis for Early Diagnosis of Malignant Lung Nodules
}

Ayman El-Baz ${ }^{1, \star}$, Matthew Nitzken ${ }^{1}$, Ahmed Elnakib ${ }^{1}$, Fahmi Khalifa ${ }^{1}$, Georgy Gimel'farb ${ }^{2}$, Robert Falk ${ }^{3}$, and Mohamed Abou El-Ghar ${ }^{4}$

${ }^{1}$ Bioimaging Laboratory, Bioengineering Department, University of Louisville, Louisville, KY, USA

2 Department of Computer Science, University of Auckland, Auckland, New Zealand

3 Department of Radiology, Jewish Hospital, Louisville, KY, USA

4 Urology and Nephrology Department, University of Mansoura, Mansoura, Egypt

\begin{abstract}
An alternative method of diagnosing malignant lung nodules by their shape, rather than conventional growth rate, is proposed. The 3D surfaces of the detected lung nodules are delineated by spherical harmonic analysis that represents a $3 \mathrm{D}$ surface of the lung nodule supported by the unit sphere with a linear combination of special basis functions, called Spherical Harmonics (SHs). The proposed 3D shape analysis is carried out in five steps: (i) 3D lung nodule segmentation with a deformable 3D boundary controlled by a new prior visual appearance model; (ii) 3D Delaunay triangulation to construct a 3D mesh model of the segmented lung nodule surface; (iii) mapping this model to the unit sphere; $(i v)$ computing the SHs for the surface; and $(v)$ determining the number of the SHs to delineate the lung nodule. We describe the lung nodule shape complexity with a new shape index, the estimated number of the SHs, and use it for the $K$-nearest classification into malignant and benign lung nodules. Preliminary experiments on 327 lung nodules (153 malignant and 174 benign) resulted in a classification accuracy of $93.6 \%$, showing that the proposed method is a promising supplement to current technologies for the early diagnosis of lung cancer.
\end{abstract}

\section{Introduction}

A great deal of work has been published regarding the usefulness of morphologic features for discriminating malignant from benign pulmonary nodules on Computed Tomography (CT) and to a lesser extent, chest radiographs. Several studies have shown a correlation between different nodule shape characteristics and underlying pathology. For example, Furuya et al. [1] analyzed the margin characteristics of 193 pulmonary nodules on high-resolution CT and subjectively classified them as one of several types, including round, lobulated, densely spiculated, ragged, and halo. They found a high level of malignancy among the lobulated $(82 \%)$, spiculated $(97 \%)$, ragged $(93 \%)$, and halo nodules $(100 \%)$, while $66 \%$ of the round nodules proved to be benign. Automatically extracted features have

\footnotetext{
* Corresponding author:- Tel:(502)-852-5092, E-mail: aselba01@louisville.edu
} 
also been shown to correlate with underlying malignancy. Kawata et al. 2] quantified the surface curvature and the degree of surrounding radiating pattern in biopsy-proven benign and malignant nodules, and compared the resulting feature maps. Their results showed good separation of the feature maps between the two categories. Similarly, fractal analysis has been used to quantify the nodule margin characteristics of benign and malignant nodules. Although none of these studies directly assessed the accuracy of their methods in predicting a diagnosis, they support the notion that nodule shape can potentially be used by automated systems to distinguish benign from malignant nodules. In summary, the existing shapebased approaches show the following limitations: $(i)$ most of them classify the lung nodules based on extracted 2D features (e.g., round, lobulated, ragged, and halo, etc.) and they did not take into account the 3D features of lung nodules; ( $i$ ) most of them did not provide a quantitative measure that has the ability to describe the shape complexity of detected lung nodules; and ( $i i i)$ most of the existing features (e.g., curvature, round, etc.) depend on the accuracy of the used nodule segmentation algorithm which make this process difficult for clinical practitioners to use. This work aims to address these limitations in a way that will make evaluating small lung masses more consistent.

\section{Methods}

\subsection{Lung Nodules Segmentation}

Accurate lung nodule segmentations from 3D Low Dose Computed Tomography (LDCT) images are a challenging problem because the intensities of the lung nodules and their surrounding tissues (e.g., blood vessels, etc.) are not clearly distinguishable. To overcome this problem, we use a conventional 3D parametric deformable boundary 3 and control its evolution with a new prior probabilistic visual appearance model. The prior is a 3D Markov-Gibbs Random Field (MGRF) model of the lung nodule intensities with translation- and rotationinvariant pairwise voxel interaction.

Let $(x, y, z)$ be Cartesian $3 \mathrm{D}$ point coordinates. A parametric deformable surface, $\mathbf{B}\left(\mathbf{P}_{1}, \ldots, \mathbf{P}_{K}\right)$, specified by $K$ control vertices, $\mathbf{P}_{k}=\left(x_{k}, y_{k}, z_{k}\right)$, evolves in the directions that minimize its energy, $E$, depending on internal, $\zeta_{\text {int }}(\mathbf{B})$, and external, $\zeta_{\text {ext }}(\mathbf{B})$, forces 3$]$ :

$$
E=E_{\text {int }}+E_{\text {ext }} \equiv \int_{\mathbf{B}}\left(\zeta_{\text {int }}(\mathbf{B})+\zeta_{\text {ext }}(\mathbf{B})\right) d \mathbf{B}
$$

In this paper, we introduce a new type of external energy that depends on the learned prior appearance model. Let $\mathbf{Q}=\{0,1, \ldots, Q-1\}$ and $\mathbf{L}=\{\mathrm{nl}$, bg $\}$ be finite sets of image intensities (gray values) and region labels, respectively. Let a finite $3 \mathrm{D}$ arithmetic lattice $\mathbf{R}=[(x, y, z): x=0, \ldots, X-1 ; y=0, \ldots, Y-1, z=$ $1, \ldots, Z-1]$ support a $3 \mathrm{D}$ image $g: \mathbf{R} \rightarrow \mathbf{Q}$ and its region map $m: \mathbf{R} \rightarrow \mathbf{L}$. The label, $m_{x, y, z}$, associates the voxel, $g_{x, y, z}$, with the lung nodule or the background. To reduce the impacts of global contrast and offset deviations of intensities due 
to different sensors, each input 3D image is normalized by mapping its signal range $\left[q_{\min }, q_{\max }\right]$ to the maximal range of $[0,255]$.

To consider the normalized images as samples of a prior MGRF model but exclude any image alignment before the segmentation, we use a generic translationand rotation-invariant MGRF with only voxel-wise and central-symmetric pairwise voxel interaction. The latter is specified by a set $\mathbf{N}$ of characteristic centralsymmetric voxel neighborhoods $\left\{\mathbf{n}_{\nu}: \nu \in \mathbf{N}\right\}$ on $\mathbf{R}$ and a corresponding set $\mathbf{V}$ of Gibbs potentials, one per neighborhood. A central-symmetric neighborhood $\mathbf{n}_{\nu}$ embraces all voxel pairs such that the $(x, y, z)$-coordinate offsets between any voxel $(x, y, z)$ and its neighbor $\left(x^{\prime}, y^{\prime}, z^{\prime}\right)$ belong to an indexed semi-open interval $\left[d_{\nu, \min }, d_{\nu, \max }\right) ; \nu \in \mathbf{N} \subset\{1,2,3, \ldots\}$ of the inter-voxel distances: $d_{\nu, \min } \leq$ $\sqrt{\left(x-x^{\prime}\right)^{2}+\left(y-y^{\prime}\right)^{2}+\left(z-z^{\prime}\right)^{2}}<d_{\nu, \max }$.

Learning the appearance prior. Let $\mathbf{S}=\left\{\left(g_{t} . m_{t}\right): t=1, \ldots, T\right\}$ be a training set of $3 \mathrm{D}$ images with known region maps. Let $\mathbf{R}_{t}=\{(x, y, z):(x, y, z) \in$ $\left.\mathbf{R} \wedge m_{t ; x, y, z}=\mathrm{nl}\right\}$ denote the part of $\mathbf{R}$ supporting lung nodule in the $t$-th training pair $\left(g_{t}, m_{t}\right) ; t=1, \ldots, T$. Let $\mathbf{C}_{\nu, t}$ be a family of voxel pairs in $\mathbf{R}_{t}^{2}$ with the co-ordinate offset $(\xi, \eta, \gamma) \in \mathbf{n}_{\nu}$ in a particular neighborhood. Let $\mathbf{F}_{\mathrm{vx}, t}$ and $\mathbf{F}_{\nu, t}$ be an empirical marginal probability distribution of voxel intensities and of intensity co-occurrences, respectively, in the training lung nodule from $\mathbf{g}_{t}: \mathbf{F}_{\mathrm{vx}, t}=$ $\left[f_{\mathrm{vx}, t}(q)=\frac{\left|\mathbf{R}_{t, q}\right|}{\left|\mathbf{R}_{t}\right|}: q \in \mathbf{Q}\right]$ and $\mathbf{F}_{\nu, t}=\left[f_{\nu, t}\left(q, q^{\prime}\right)=\frac{\left|\mathbf{C}_{\nu, t ; q, q^{\prime}}\right|}{\left|\mathbf{C}_{\nu, t}\right|}:\left(q, q^{\prime}\right) \in \mathbf{Q}^{2}\right]$ where $\mathbf{R}_{t, q}=\left\{(x, y, z):(x, y, z) \in \mathbf{R}_{t} \wedge g_{x, y, z}=q\right\}$ is a subset of voxels supporting the intensity $q$ and $\mathbf{C}_{\nu, t ; q, q^{\prime}}$ is a subset of the voxel pairs $\mathbf{c}_{\xi, \eta, \gamma}(x, y, z)=$ $((x, y, z),(x+\xi, y+\eta, z+\gamma)) \in \mathbf{R}_{t}^{2}$ supporting the intensity co-occurrence $\left(q, q^{\prime}\right)$ in the training lung nodule from $\mathbf{g}_{t}$. Let $\mathbf{V}_{\mathrm{vx}}=\left[V_{\mathrm{vx}}(q): q \in \mathbf{Q}\right]$ be a potential function of voxel intensities that describes the voxel-wise interaction. Let $\mathbf{V}_{\nu}=\left[V_{\nu}\left(q, q^{\prime}\right):\left(q, q^{\prime}\right) \in \mathbf{Q}^{2}\right]$ be a potential function of intensity co-occurrences in the neighboring voxel pairs that describes the pairwise interaction in the neighborhood $\mathbf{n}_{\nu} ; \nu \in \mathbf{N}$. The MGRF model of the $t$-th training pair is specified by the joint Gibbs probability distribution on the sublattice $\mathbf{R}_{t}$ :

$$
P_{t}=\frac{1}{Z_{t}} \exp \left(\left|\mathbf{R}_{t}\right|\left(\mathbf{V}_{\mathrm{vx}}^{\top} \mathbf{F}_{\mathrm{vx}, t}+\sum_{\nu \in \mathbf{N}} \rho_{\nu, t} \mathbf{V}_{\nu, t}^{\top} \mathbf{F}_{\nu, t}\right)\right)
$$

where $\rho_{\nu, t}=\left|\mathbf{C}_{\nu, t}\right| /\left|\mathbf{R}_{t}\right|$ is the average cardinality of $\mathbf{n}_{\nu}$ with respect to $\mathbf{R}_{t}$.

To identify the MGRF model in Eq. (2), the Gibbs potentials are approximated analytically 1 :

$$
\begin{array}{cc}
V_{\mathrm{vx}, \mathrm{nl}}(q)=\log f_{\mathrm{vx}, \mathrm{nl}}(q)-\frac{1}{Q} \sum_{\kappa \in \mathbf{Q}} \log f_{\mathrm{vx}, \mathrm{nl}}(\kappa) & \text { for } q \in \mathbf{Q} ; \text { and } \\
V_{\nu, \mathrm{nl}}\left(q, q^{\prime}\right)=\lambda \rho_{\nu}\left(f_{\nu, \mathrm{nl}}\left(q, q^{\prime}\right)-f_{\mathrm{vx}, \mathrm{nl}}(q) f_{\mathrm{vx}, \mathrm{nl}}\left(q^{\prime}\right)\right) & \text { for }\left(q, q^{\prime}\right) \in \mathbf{Q}^{2}
\end{array}
$$

where the common factor $\lambda$ is also computed analytically.

\footnotetext{
${ }^{1}$ For proof, please see: https://louisville.edu/speed/bioengineering/faculty/ bioengineering-full/dr-ayman-el-baz/supplemental-materials.
} 
Boundary evolution under the appearance models. To guide the boundary evolution, we embed in the external energy term of Eq. (11) the learned prior appearance model of the detected lung nodule as follows:

$$
\zeta_{\text {ext }}(\mathbf{P}=(x, y, z))=-\pi_{\mathbf{p}}\left(g_{x, y, z} \mid \mathbf{S}\right)
$$

Here, $\pi_{\mathbf{p}}(q \mid \mathbf{S})$ is the prior conditional probability of $q$, given the fixed current intensities in the characteristic central-symmetric neighborhood of $\mathbf{P}$ for the MGRF model of Eq. (2):

$$
\pi_{\mathbf{P}}\left(g_{x, y, z} \mid \mathbf{S}\right)=\exp \left(E_{\mathbf{P}}\left(g_{x, y, z} \mid \mathbf{S}\right)\right) / \sum_{q \in \mathbf{Q}} \exp \left(E_{\mathbf{P}}(q \mid \mathbf{S})\right)
$$

where $E_{\mathbf{P}}(q \mid \mathbf{S})$ is the conditional Gibbs energy of pairwise interaction for the voxel $\mathbf{P}$ provided that an intensity $q$ is assigned to the lung nodule while the other current intensities in all its neighboring voxels over the characteristic neighborhoods $\mathbf{n}_{\nu} ; \nu \in \mathbf{N}$, remains fixed:

$$
E_{\mathbf{P}}(q \mid \mathbf{S})=V_{\mathrm{vx}, \mathrm{nl}}(q)+\sum_{\nu \in \mathbf{N}} \sum_{(\xi, \eta, \gamma) \in \mathbf{n}_{\nu}}\left(V_{\nu, \mathrm{nl}}\left(g_{x-\xi, y-\eta, z-\gamma}, q\right)+V_{\nu, \mathrm{nl}}\left(q, g_{x+\xi, y+\eta, z+\gamma}\right)\right)
$$

After changing the energy $E_{\mathbf{B}}$ of the $3 \mathrm{D}$ region $\mathbf{R}_{\mathbf{B}} \subset \mathbf{R}$ inside the evolving boundary B:

$$
E_{\mathbf{B}}=\sum_{\forall \mathbf{P}=(x, y, z) \in \mathbf{R}_{\mathbf{B}}} E_{\mathbf{P}}\left(g_{x, y, z} \mid \mathbf{S}\right)
$$

stops, the evolution terminates.

\subsection{Spherical Harmonics (SHs) Shape Analysis}

Spectral SH analysis [4]5] considers 3D surface data as a linear combination of specific basis functions. In our case, the surface of the segmented lung nodule is first approximated by a triangulated 3D mesh (see Fig. 1) built with an algorithm by Fang and Boas [6. Secondly, the lung nodule surface for each subject is mapped for the SH decomposition to the unit sphere. We propose a novel mapping approach, called "Attraction-Repulsion," that calls for all the mesh nodes to meet two conditions: $(i)$ the unit distance of each node from the lung nodule center, and (ii) an equal distance of each node from all of its nearest neighbors.

To detail our Attraction-Repulsion algorithm (see its summary in Algorithm 1), let $\tau$ denote the iteration index, $I$ be the total number of the mesh nodes (in all the experiments below $I=4896$ nodes), and $\mathbf{P}_{\tau, i}$ be the Cartesian coordinates of the surface node $i$ at iteration $\tau ; i=1, \ldots, I$. Let $J$ be the number of the neighbors for a mesh node and $d_{\tau, i j}$ denote the Euclidean distance between the surface nodes $i$ and $j$ at iteration $\tau$, where $i=1, \ldots, I$ and $j=1, \ldots, J$. Let $\boldsymbol{\Delta}_{\tau, j i}=\mathbf{P}_{\tau, j}-\mathbf{P}_{\tau, i}$ denote the displacement between the nodes $j$ and $i$ at iteration $\tau$. Let $C_{\mathrm{A}, 1}, C_{\mathrm{A}, 2}$, and $C_{\mathrm{R}}$ be the attraction and repulsion constants, respectively, that control the displacement of each surface node. 

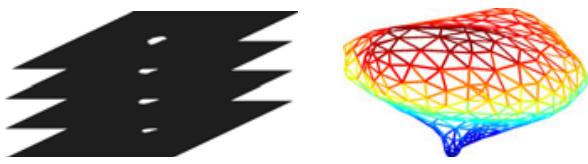

Fig. 1. Generating a 3D mesh for the lung nodule surface from a stack of successive segmented 2D LDCT slices
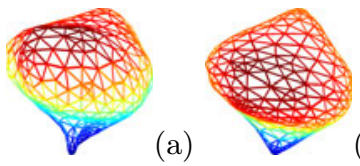

(b)

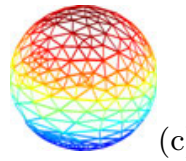

(c)

The starting attraction step of the proposed mapping tends to center each node, $\mathbf{P}_{i} ; i=1, \ldots, I$, with respect to its neighbors by iteratively adjusting its location:

$$
\mathbf{P}_{\tau, i}^{\prime}=\mathbf{P}_{\tau, i}+C_{\mathrm{A}, 1} \sum_{j=1 ; j \neq i}^{J} \boldsymbol{\Delta}_{\tau, j i} d_{\tau, j i}^{2}+C_{\mathrm{A}, 2} \frac{\boldsymbol{\Delta}_{\tau, j i}}{d_{\tau, j i}}
$$

where the factor $C_{\mathrm{A}, 2}$ keeps the tightly packed nodes from collision and pushes the adjusted nodes away from their neighbors if a certain neighbor is much closer than the others.

The subsequent repulsion step inflates the whole mesh by pushing all the nodes outwards to become evenly spaced after their final back-projection onto the unit sphere along the rays from the center of the sphere. To ensure that the nodes that have not been shifted will not collide with the altered node, the location of each node, $\mathbf{P}_{i} ; i=1, \ldots, I$, is updated before the back-projection as:

$$
\mathbf{P}_{\tau+1, i}^{\circ}=\mathbf{P}_{\tau, i}^{\prime}+\frac{C_{\mathrm{R}}}{2 I} \sum_{j=1 ; j \neq i}^{I}\left(\frac{\boldsymbol{\Delta}_{\tau, j i}}{\left|\boldsymbol{\Delta}_{\tau, j i}\right|^{2}}\right)
$$

where a repulsion constant $C_{\mathrm{R}}$ controls the displacement of each surface node and establishes a balance between the processing time and accuracy (e.g., a smaller $C_{\mathrm{R}}$ values guarantees that the node faces will not become crossed during the iterations at the expense of the increased processing time). All the experiments below are obtained with $0.3 \leq C_{\mathrm{R}} \leq 0.7$.

The original lung nodule mapped to the unit sphere with the proposed Attraction-Repulsion algorithm is approximated by a linear combination of SHs, the lower-order harmonics being sufficient to represent more generic information, while the finer details requiring the higher-order ones. The SHs are generated by solving an isotropic heat equation for the nodule surface on the unit sphere. Let $\mathcal{S}: \mathbf{M} \rightarrow \mathbf{U}$ denote the mapping of a nodule mesh $\mathbf{M}$ to the unit sphere $\mathbf{U}$. Each node $\mathbf{P}=(x, y, z) \in \mathbf{M}$ mapped to the spherical position $\mathbf{u}=S(\mathbf{P})$ is represented by the spherical coordinates $\mathbf{u}=(\sin \theta \cos \varphi, \sin \theta \sin \varphi, \cos \theta)$ where $\theta \in[0, \pi]$ and $\varphi \in[0,2 \pi)$ are the polar and azimuth angles, respectively. The $\mathrm{SH}$ $Y_{\alpha \beta}$ of degree $\alpha$ and order $\beta$ is defined as [7]: 


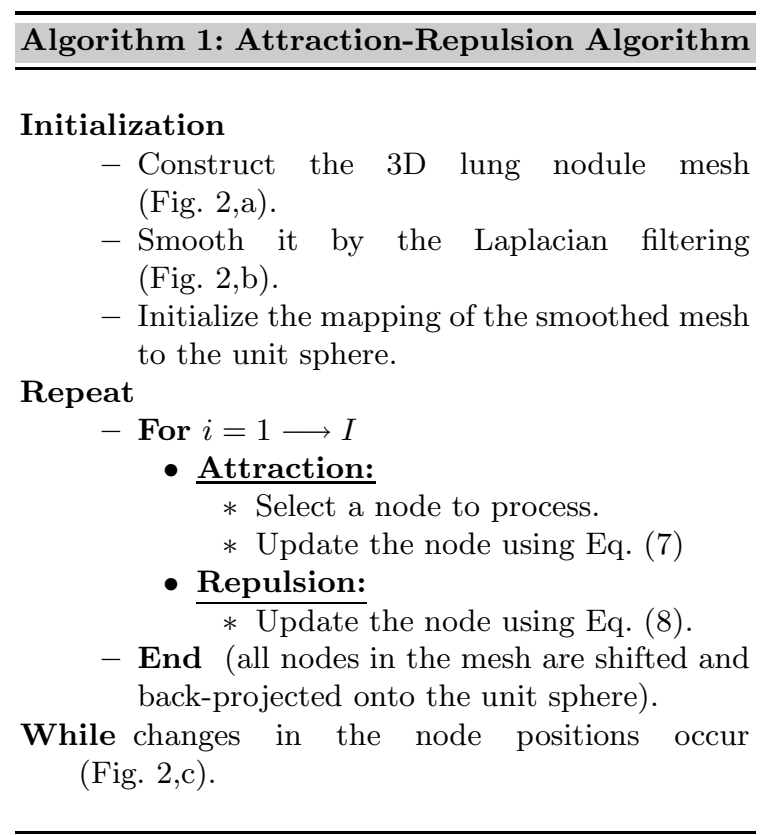

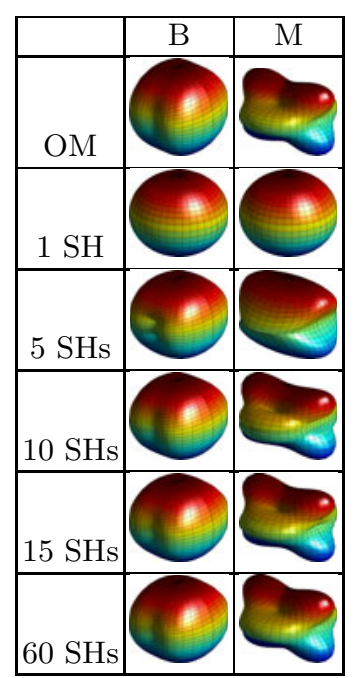

Fig. 3. Approximation of the $3 \mathrm{D}$ shape for malignant (M), benign nodules (B), and original mesh (OM)

$$
Y_{\alpha \beta}=\left\{\begin{array}{lr}
c_{\alpha \beta} G_{\alpha}^{|\beta|} \cos \theta \sin (|\beta| \varphi) & -\alpha \leq \beta \leq-1 \\
\frac{c_{\alpha \beta}}{\sqrt{2}} G_{\alpha}^{|\beta|} \cos \theta & \beta=0 \\
c_{\alpha \beta} G_{\alpha}^{|\beta|} \cos \theta \cos (|\beta| \varphi) & 1 \leq \beta \leq \alpha
\end{array}\right.
$$

where $c_{\alpha \beta}=\left(\frac{2 \alpha+1}{2 \pi} \frac{(\alpha-|\beta|) !}{(\alpha+|\beta|) !}\right)^{\frac{1}{2}}$ and $G_{\alpha}^{|\beta|}$ is the associated Legendre polynomial of degree $\alpha$ and order $\beta$. For a fixed $\alpha$, the polynomials $G_{\alpha}^{\beta}$ are orthogonal over the range $[-1,1]$. As shown in [7, the Legendre polynomials are effective in calculating SHs. This is the main motivation behind their use in this work.

Finally, the lung nodule is reconstructed from the SHs of Eq. (9). In the case of the SHs expansion, the standard least-square fitting does not accurately model the $3 \mathrm{D}$ shape of the lung nodule and can miss some of the shape details that discriminate between the malignant and benign lung nodules. To circumvent this problem, we used the iterative residual fitting by Shen et al. [8] that accurately approximates the 3D shapes of malignant and benign lung nodules. As shown in Fig. 3. the model accuracy does not significantly change for the benign nodule from 15 to $60 \mathrm{SHs}$, while it continues to increase for the malignant nodule.

\subsection{Quantitative Lung Nodule Shape Analysis}

Our main hypothesis is that the shape of malignant nodules is more complicated (e.g., with spiculation) when compared with the shape of benign nodules which are simpler (smoothed shape) as in Fig. 3. so that more SHs have to be used for 


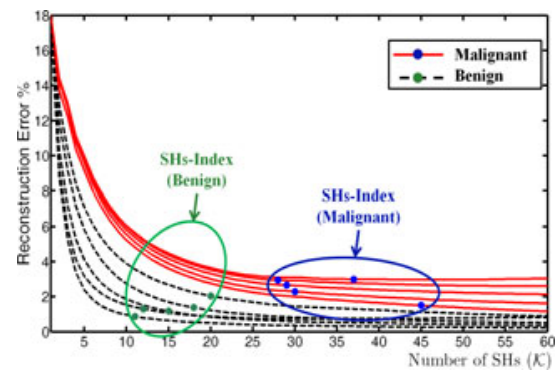

Fig. 4. Estimation of the shape index from the total nodule approximation error for malignant and benign nodules

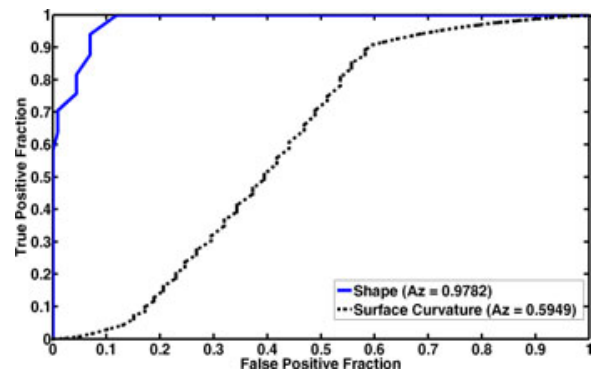

Fig. 5. The ROC curves for our approach and the surface curvature-based diagnostic approach

accurate approximation of the shape of malignant lung nodule. Therefore, the number of the SHs after which there are no significant changes in the approximations can be used as a new shape index that quantifies the shape complexity of the detected lung nodules. Due to the unit sphere mapping, the original mesh for each nodule is inherently aligned with its reconstructed mesh shape, and the sum of the Euclidean distances between the corresponding nodes gives the total error between both the mesh models. As shown in Fig. 4 the total error curves for the increasing number $\mathcal{K}$ of the $\mathrm{SHs}$ can be statistically analyzed to differentiate between the detected lung nodules.

\section{Experimental Results and Conclusions}

To justify the proposed methodology of analyzing the 3D shape of both malignant and benign nodules, the above proposed shape analysis framework was pilot-tested on a database of clinical multislice chest LDCT scans of 327 lung nodules (153 malignant and 174 benign). The $\mathrm{CT}$ data sets each have $0.7 \times 0.7 \times$ $2.0 \mathrm{~mm}^{3}$ voxels, with nodule diameters ranging from $3 \mathrm{~mm}$ to $30 \mathrm{~mm}$. Note that these 327 nodules were diagnosed using either bronchoscopy and needle biopsy, or two-year follow-up with CT scans2. Also, our current database does not contain Ground Glass Nodules (GGN).

The training subset for classification (15 malignant lung nodules and 15 benign lung nodules) were arbitrarily selected from 327 lung nodules. The accuracy of classification based on using a K-nearest classifier for both the training and test subjects was evaluated using the $\chi^{2}$-test at $95 \%$ confidence level. At the $95 \%$ confidence level, 143 out of 153 malignant nodules (a $93.5 \%$ accuracy) were correctly classified, and 163 out of 174 control subjects (a $93.7 \%$ accuracy) were correctly classified. The overall accuracy using the proposed 3D shape-based CAD system is $93.6 \%$ in the first detection of lung nodules. The classification based on the

\footnotetext{
${ }^{2}$ For complete details about our nodules database, please see: https://louisville. edu/speed/bioengineering/faculty/bioengineering-full/dr-ayman-el-baz/ supplemental-materials.
} 
traditional analysis of surface curvature-based diagnostic approach [2] correctly classifies 77 out of 153 malignant nodules (a $50.3 \%$ accuracy), and 103 out of 174 benign nodules (a $59.2 \%$ accuracy) at a $95 \%$ confidence level. These results highlight the advantage of the proposed approach.

Another way to measure and test the performance of the proposed diagnostic system is to compute the Receiver Operating Characteristic (ROC). Each point on the graph is generated by using a different cut point (classification threshold). Figure 5 shows the ROC curves of the two approaches, our proposed shape index-based diagnostic approach and the surface curvature-based diagnostic approach [2]. It is clear from the data in Fig. [5 that the area under the ROC curve of our present approach is much larger $(\mathrm{Az}=0.9782)$ than the area under the ROC curve of the surface curvature-based diagnostic approach 2 ( $\mathrm{Az}=0.5949)$. The high sensitivity and specificity of the proposed approach is due to using the estimated number of spherical harmonics to approximate the 3D shape of a detected lung nodule as a new discriminatory feature which is more separable than using surface curvature. More experimental results that address the sensitivity of our approach w.r.t. the accuracy of segmentation and mesh generation steps has been posted on our web site ${ }^{2}$.

As demonstrated in this paper, the preliminary results justify the elaboration of the proposed alternative method for diagnosing malignant lung nodules. Its novelty lies in using the shape of a $3 \mathrm{D}$ nodule instead of the more conventional surface curvature as a reliable diagnostic feature. The shape is described in terms of a linear combination of SHs.

\section{References}

1. Furuya, K., Murayama, S., Soeda, H., Murakami, J., Ichinose, Y., Yabuuchi, H., Katsuda, Y., Koga, M., Masuda, K.: New Classification of Small Pulmonary Nodules by Margin Characteristics on High-resolution CT. Acta Radiol. 40(5), 496-504 (1999)

2. Kawata, Y., Niki, N., Ohmatsu, H., Kakinuma, R., Eguchi, K., Kaneko, R., Moriyama, N.: Quantitative Surface Characterization of Pulmonary Nodules based on Thin-section CT Images. IEEE Trans. Nucl. Sci. 45(4), 2132-2138 (1998)

3. Kass, M., Witkin, A., Terzopoulos, D.: Snakes: Active Contour Models. Int. J. Comput. Vision 1(4), 321-331 (1987)

4. Chung, M.K., Evans, A.C., Davidson, R.J.: Weighted Fourier Series Representation and its Application to Quantifying the Amount of Gray Matter. IEEE Trans. Med. Imag. 26(4), 566-581 (2007)

5. Nain, D., Haker, S., Bobick, A., Tannenbaum, A.: Multiscale 3D Shape Analysis using Spherical Wavelets. In: Duncan, J.S., Gerig, G. (eds.) MICCAI 2005. LNCS, vol. 3750, pp. 459-467. Springer, Heidelberg (2005)

6. Fang, Q., Boas, D.: Tetrahedral Mesh Generation from Volumetric Binary and Grayscale Images. In: 6th IEEE International Symposium on Biomedical Imaging: From Nano to Macro (ISBI 2009), pp. 1142-1145 (2009)

7. Courant, R., Hilbert, D.: Methods of Mathematical Physics. John Willy\&Sons, New York (1989)

8. Shen, L., Chung, M.K.: Large-scale Modeling of Parametric Surfaces using Spherical Harmonics. In: 3rd International Symposium on 3D Data Processing, Visualization, and Transmission (3DPVT 2006), pp. 294-301 (2006) 